\title{
Cosmological evolution in compactified Hořava-Witten theory induced by matter on the branes
}

\author{
Ulrich Ellwanger \\ Laboratoire de Physique Théorique \\ Université de Paris XI, Bâtiment 210, F-91405 ORSAY Cedex, France
}

\begin{abstract}
The combined Einstein equations and scalar equation of motion in the Hořava-Witten scenario of the strongly coupled heterotic string compactified on a Calabi-Yau manifold are solved in the presence of additional matter densities on the branes. We take into account the universal Calabi-Yau modulus $\varphi$ with potentials in the 5- $d$ bulk and on the 3-branes, and allow for an arbitrary coupling of the additional matter to $\varphi$ and an arbitrary equation of state. No ad hoc stabilization of the five dimensional radius is assumed. The matter densities are assumed to be small compared to the potential for $\varphi$ on the branes; in this approximation we find solutions in the bulk which are exact in $y$ and t. Depending on the coupling of the matter to $\varphi$ and its equation of state, various solutions for the metric on the branes and in the 5 - $d$ bulk are obtained: Solutions corresponding to a "rolling radius", and solutions with a static 5- $d$ radius, which reproduce the standard cosmological evolution.
\end{abstract}

LPT Orsay 00-02

January 2000

*Unité Mixte de Recherche - CNRS - UMR 8627 


\section{Introduction}

Motivated, to a large extent, by the Hořava-Witten scenario of the strongly coupled heterotic string [1], the cosmology of a five dimensional universe bounded by 3-branes has recently been the subject of many investigations. If one estimates the parameters in the Hořava-Witten scenario using, as input, the phenomenological values of Newton's constant, $M_{G U T}$ and $\alpha_{G U T}$ [2, 3] one finds that there exists a regime below $M_{G U T}$ (the inverse size of a Calabi-Yau manifold, on which 6 dimensions are compactified) in which a five dimensional description of our universe is appropriate.

The topology of the five dimensional universe is the one of a $S^{1} / Z_{2}$ orbifold: There are two distinct 3-branes, and the fields in the bulk between the branes are either symmetric or anti-symmetric with respect to $Z_{2}$, i.e. reflections at the branes. The field content in the five dimensional bulk is the one of $N=1$ (gauged) supergravity, plus "matter" originating from a 3-form in 11 dimensions, and from internal degrees of freedom of the metric (moduli) [4-7]. On the branes we have, in addition, matter originating from $E_{8}$ Yang-Mills theories in 10 dimensions.

Solutions to the equations of motion in the five dimensional bulk have been obtained in $[4-12]$. Due to the presence of potentials for the moduli on the branes (with opposite signs) one finds that the moduli configurations vary with $y$, the fifth coordinate, leading to a $y$-dependent energy-momentum tensor in the bulk.

Independently from the Hořava-Witten theory, scenarios for the cosmology of a five dimensional universe with branes have been developed. Assuming a $y$-independent cosmological constant in the bulk, and fine-tuned cosmological constants on the branes (with opposite signs), Randall and Sundrum [13 (RSI) found a static solution of the Einstein equations in the bulk with an exponential dependence of the metric on $y$. They argued that this phenomenon could solve the hierachy problem, provided our four dimensional universe lives on a brane with negative tension (negative cosmological constant). Alternatively, if we live on a brane with positive tension surrounded by a bulk with a $y$-independent cosmological constant [14, 15] (RSII), the physical distance between the branes may go off to infinity without affecting the validity of Newton's law on our brane (due to additional massless graviton Kaluza-Klein modes).

Interestingly, once one studies the cosmological evolution induced by additional matter on the branes, the conventional (and successful) relation $3 H^{2}=8 \pi G_{N} \rho$ 
between the Hubble parameter $H$, Newton's constant $G_{N}$ and the energy density $\rho$ is not always obtained. Consequently the (non)-validity of this relation, at least at temperatures below $\sim 1 \mathrm{MeV}$, serves to eliminate certain scenarios.

First, the cosmological evolution induced by matter on the branes has been studied in the simplest case of vanishing cosmological constants in the bulk and on the branes by Binétruy, Deffayet and Langlois in [16]. The astonishing result was that the conventional relation $H^{2} \sim \rho$ does not hold, rather one obtains $H \sim \rho$ (in contradiction, e.g., with the otherwise successful big bang nucleo-synthesis).

Subsequently, the matter-induced cosmological evolution in the RSI scenario was investigated in [17, 18] with the "negative" result $H^{2} \sim-\rho$ (if we live on the brane with negative tension, which is a necessary condition for the solution of the hierarchy problem in this case).

On the other hand, the cosmological evolution induced by matter on a brane with positive tension agrees with the conventional evolution (at late times) [17 23 if one assumes that the physical distance between the branes is stabilized, and the relative values of the cosmological constants in the bulk and on the branes are fine-tuned.

The purpose of the present paper is the study, in the (compactified) HorravaWitten theory, of the cosmological evolution induced by matter on the branes. We allow for an arbitrary equation of state, i.e., a relation $p=w \rho$ among the pressure $p$ and the energy density $\rho$. As fields in the bulk we consider, apart from the components of the graviton, a scalar field $\varphi$ which corresponds to the universal Calabi-Yau modulus. The presence of this field is model independent and, due to the non-vanishing "internal" components of the field strength associated to the 3form, one obtains potentials $V(\varphi)$ both in the bulk and on the branes [4-7]. If one introduces a dimensionful parameter $\gamma$ with $\gamma \sim \mathcal{O}\left(M_{G U T}^{4} / M_{11}^{3}\right)$, where $M_{11}$ is scale of the gravitational coupling in eleven dimensions, one finds for the potential in the bulk $V^{(b u l k)}(\varphi) \sim \mathcal{O}\left(\gamma^{2}\right)$, whereas the potentials $V^{(n)}$ on the branes satisfy $V^{(n)}(\varphi) \sim \mathcal{O}(\gamma)$.

Subsequently we will assume $\rho, p \ll \gamma \kappa_{5}^{-2}$ where $\kappa_{5}^{2}$ is the inverse gravitational coupling in five dimensions with mass dimension $\kappa_{5}^{2}=M_{5}^{-3}$. This assumption is certainly realistic with respect to "Standard Model" matter on our brane, but our subsequent results will also apply to "non-standard" matter (e.g. associated with gaugino condensation on the hidden brane) as long as the above inequality is satisfied. Notably we will allow for an arbitrary dependence of the "matter" Lagrangians 
on the branes, $\mathcal{L}^{(n)}$, on the field $\varphi: \mathcal{L}^{(n)}=\mathcal{L}^{(n)}(\varphi)\left(\right.$ with $\left.\mathcal{L}^{(n)} \sim \mathcal{O}(\rho, p) \ll \mathcal{O}\left(\gamma \kappa_{5}^{-2}\right)\right)$ which affects the junction conditions of the field $\varphi$ on the branes, see below.

The inflationary evolution in this set-up, induced by matter potentials on the branes (corresponding to an equation of state $p=\rho$ ), has previously been discussed in [24]. The results obtained in [24] correspond either to the case $\gamma \cdot R_{5} \ll 1$, which allows for a linearized approximation of the $y$ dependence of the fields, or to "matter" Lagrangians $\mathcal{L}^{(n)}$ on the branes which are $\varphi$ independent.

Below, we will construct solutions in the bulk which are exact in $y$, since we will not assume $\gamma \cdot R_{5} \ll 1$. Notably, we will not assume that the physical distance between the branes (or the $y y$-component of the metric in the bulk) is stabilized in an ad hoc fashion (corresponding mechanisms are discussed in [23, 25 - 30]): One of our aims is to see, under which general circumstances solutions with a static size of the fifth dimension exist. In fact we will obtain, depending on the $\varphi$ dependence of $\mathcal{L}^{(n)}(\varphi)$, both solutions corresponding to a "rolling radius", as well as solutions with a static $y y$-component of the metric. Not astonishingly, only the latter case allows for a conventional cosmological evolution on a brane.

In the next section we will present the general set-up of our approach: the action in the bulk and on the branes, the Einstein equations and the equation of motion of $\varphi$, the junction conditions on the branes, and the exact (static) solution of [4. In section 3 we introduce our ansatz for the time-dependent fields in the bulk, which is motivated by the search for a conventional cosmological evolution on the branes: we will assume, that the non-trivial time-dependence is induced by the presence of matter on the branes. A self-consistent ansatz is seen to be $\partial_{t} \sim \mathcal{O}\left(\left(\rho \gamma \kappa_{5}^{2}\right)^{1 / 2}\right)$, where $\partial_{t}$ denotes the time derivative of any field in the bulk or on the branes. (Given the existence of time dependent solutions with $\partial_{t} \sim \mathcal{O}(\gamma)$ [9, 10], this ansatz is certainly not the most general one. It represents, on the other hand, the "minimal" time dependence which is induced by the presence of matter on the branes). Then the Einstein equations and the equation of motion of $\varphi$ can be solved exactly in $y$ and $t$, but neglecting terms of relative order $\rho \kappa_{5}^{2} \gamma^{-1}$. In section 4 we discuss the physical properties of our solutions and conclude.

\section{Equations of motion and junction conditions}


Our starting point is a five dimensional action in the bulk which depends, apart from the gravitational sector, on a scalar field $\varphi$ which is related to the universal modulus of the internal Calabi-Yau manifold $[4-7]$. In the notation of [4, our field $\varphi$ is related to the field $V$ in [⿴囗⿰丿㇄口] by $V=\exp (2 \varphi)$. Likewise, our parameter $\gamma$ appearing in the potentials of $\varphi$ in the bulk and on the branes is related to the parameter $\alpha$ in 四 through $\gamma=\sqrt{2} \alpha$. In any case $\gamma$ is of $\mathcal{O}\left(M_{G U T}^{4} / M_{11}^{3}\right)$. The exact value of $\gamma$ depends on the shape of the Calabi-Yau manifold [2, 国. The bulk action then reads

$$
S^{\text {bulk }}=-\frac{1}{\kappa_{5}^{2}} \int \sqrt{-g_{5}} d^{5} x\left\{\frac{1}{2} R+\partial_{\mu} \varphi \partial^{\mu} \varphi+\frac{\gamma^{2}}{12} e^{-4 \varphi}\right\} .
$$

Subsequently we denote the fifth dimension (across the bulk) by $y$ and assume, that all fields depend on $t$ and $y$, but not on the 3 spatial coordinates $x_{i}$. A convenient (diagonal) ansatz for the metric is then

$$
d s^{2}=-e^{2 \nu} d t^{2}+e^{2 \alpha}(d \vec{x})^{2}+e^{2 \beta} d y^{2} .
$$

Denoting time derivatives by points, and derivatives with respect to $y$ by primes, the Einstein equations in the bulk become

$$
\begin{gathered}
-e^{-2 \nu}\left(\dot{\alpha}^{2}+\dot{\alpha} \dot{\beta}\right)+e^{-2 \beta}\left(\alpha^{\prime \prime}+2 \alpha^{\prime 2}-\alpha^{\prime} \beta^{\prime}\right)=\frac{\kappa_{5}^{2}}{3} T_{0}^{0}, \\
-e^{-2 \nu}\left(2 \ddot{\alpha}+3 \dot{\alpha}^{2}-2 \dot{\alpha} \dot{\nu}+\dot{\beta}(2 \dot{\alpha}-\dot{\nu})+\ddot{\beta}+\dot{\beta}^{2}\right) \\
+e^{-2 \beta}\left(2 \alpha^{\prime \prime}+\nu^{\prime \prime}+3 \alpha^{\prime 2}+\nu^{\prime 2}+2 \alpha^{\prime} \nu^{\prime}-\beta^{\prime}\left(2 \alpha^{\prime}+\nu^{\prime}\right)\right)=\kappa_{5}^{2} T_{S}, \\
\dot{\alpha} \nu^{\prime}-\dot{\alpha}^{\prime}-\dot{\alpha} \alpha^{\prime}+\alpha^{\prime} \dot{\beta}=\frac{\kappa_{5}^{2}}{3} T_{05}, \\
-e^{-2 \nu}\left(\ddot{\alpha}+2 \dot{\alpha}^{2}-\dot{\alpha} \dot{\nu}\right)+e^{-2 \beta}\left(\alpha^{\prime 2}+\alpha^{\prime} \nu^{\prime}\right)=\frac{\kappa_{5}^{2}}{3} T_{5}^{5} .
\end{gathered}
$$

Here $T_{S}$ denotes the diagonal element of the spatial components of the energy momentum tensor,

$$
T_{i}^{j}=T_{S} \delta_{i}^{j}
$$


Given the action (2.1), and recalling that $\varphi$ depends only on $t$ and $y$, the nonvanishing components of the energy momentum tensor in the bulk (away from the branes) are

$$
\begin{gathered}
\kappa_{5}^{2} T_{0}^{0}=-e^{-2 \nu} \dot{\varphi}^{2}-e^{-2 \beta} \varphi^{\prime 2}-\frac{\gamma^{2}}{12} e^{-4 \varphi}, \\
\kappa_{5}^{2} T_{S}=e^{-2 \nu} \dot{\varphi}^{2}-e^{-2 \beta} \varphi^{\prime 2}-\frac{\gamma^{2}}{12} e^{-4 \varphi}, \\
\kappa_{5}^{2} T_{5}^{5}=e^{-2 \nu} \dot{\varphi}^{2}+e^{-2 \beta} \varphi^{\prime 2}-\frac{\gamma^{2}}{12} e^{-4 \varphi}, \\
\kappa_{5}^{2} T_{05}=2 \dot{\varphi} \varphi^{\prime} .
\end{gathered}
$$

Finally we have to consider the equation of motion of the field $\varphi$, which takes the form (away from the branes)

$$
-e^{-2 \nu}(\ddot{\varphi}+\dot{\varphi}(3 \dot{\alpha}-\dot{\nu}+\dot{\beta}))+e^{-2 \beta}\left(\varphi^{\prime \prime}+\varphi^{\prime}\left(3 \alpha^{\prime}+\nu^{\prime}-\beta^{\prime}\right)\right)=-\frac{\gamma^{2}}{6} e^{-4 \varphi} .
$$

Now we consider the actions on the branes, which are situated at $y^{(n)}=0, \pi R_{5}$. We parametrize them as

$$
S^{(n)}=\int \sqrt{-g_{4}} d^{4} x\left\{\mathcal{L}_{\text {matter }}^{(n)}(\varphi)-V^{(n)}(\varphi)\right\} .
$$

Here $\mathcal{L}_{\text {matter }}^{(n)}(\varphi)$ depends, of course, on many additional matter fields $\phi_{i}$. Below, however, only the possible dependence on the bulk field $\varphi$ will play a role. Depending on the precise form of $\mathcal{L}_{\text {matter }}^{(n)}$, and on the configurations of the fields $\phi_{i}$, this part of the brane actions will contribute to the energy momentum tensors on the branes in the form of energy densities $\rho^{(n)}$ and pressures $p^{(n)}$, see below.

The potentials $V^{(n)}(\varphi)$ on the two branes are known to be of the form [4, 5]

$$
V^{(n)}(\varphi)=\mp \frac{\gamma}{\kappa_{5}^{2}} e^{-2 \varphi}
$$

Here the minus sign applies to the brane at $y=0$, and the plus sign to the brane at $y=\pi R_{5}$. Since we assume 


$$
\mathcal{L}_{\text {matter }}^{(n)} \sim \rho^{(n)} \sim p^{(n)} \ll \frac{\gamma}{\kappa_{5}^{2}},
$$

the brane at $y=0$ is thus the brane with negative tension, if $\gamma$ is positive. We can easily interchange the role of the two branes (negative vs. positive tension) by changing the sign of $\gamma$.

As is well known, the presence of branes leads to additional singular terms (proportional to $\delta$-functions in $y$ ) on the right-hand sides of the Einstein equations (2.3a), (2.3b), and the equation of motion (2.6), which have to be matched by singularities in the second derivatives in $y$ on the left-hand side. Since all fields under consideration are symmetric under the orbifold symmetry $Z_{2}$, these jumps in the first derivatives in $y$ fix these first derivatives completely at $y^{(n)}=0, \pi R_{5}$. Here, these junction conditions read

$$
\begin{gathered}
\alpha^{\prime(n)}=\frac{\kappa_{5}^{2}}{6} e^{\beta} T_{0}^{0(n)}, \\
\nu^{\prime(n)}=\frac{\kappa_{5}^{2}}{6} e^{\beta}\left(3 T_{S}^{(n)}-2 T_{0}^{0(n)}\right), \\
\varphi^{\prime(n)}=\frac{\kappa_{5}^{2}}{4} e^{\beta} \frac{\delta}{\delta \varphi}\left(V^{(n)}(\varphi)-\mathcal{L}_{\text {matter }}^{(n)}(\varphi)\right),
\end{gathered}
$$

with

$$
\begin{gathered}
T_{0}^{0(n)}=-V^{(n)}(\varphi)-\rho^{(n)}, \\
T_{S}^{(n)}=-V^{(n)}(\varphi)+p^{(n)}
\end{gathered}
$$

and $V^{(n)}(\varphi)$ as in eq. (2.8) As discussed above, $\rho^{(n)}$ and $p^{(n)}$ originate from the matter Lagrangian on the branes, which we will not specify any further at this stage.

Static solutions to the Einstein equations (2.3), the scalar equation of motion (2.6) and the junction conditions (2.10) - in the absence of matter on the branes have been given in $\llbracket$. We will denote these solutions by $\widetilde{\alpha}, \widetilde{\nu}, \widetilde{\beta}$ and $\widetilde{\varphi}$, which read 


$$
\begin{gathered}
\widetilde{\alpha}(y)=\widetilde{\nu}(y)=\frac{1}{2} \ln H+A, \\
\widetilde{\beta}(y)=2 \ln H+B, \\
\widetilde{\varphi}(y)=\frac{3}{2} \ln H+\frac{1}{2} B, \\
H=\frac{\gamma}{3}|y|+C \quad \text { for }-\pi R_{5} \leq y \leq \pi R_{5}, \quad H\left(y+2 \pi R_{5}\right)=H(y) .
\end{gathered}
$$

$A, B$ and $C$ are arbitrary constants; in terms of a supergravity Lagrangian in four dimensions $B$ and $C$ correspond to linear combinations of the arbitrary vevs of the standard fields $\operatorname{Re}(S)$ and $\operatorname{Re}(T)$ [24].

Time dependent solutions have been obtained in [9, 10], but in the next sections we seek for cosmological solutions, where the time dependence is induced by the presence of matter on the branes. Hence the static solutions (2.12) will serve as a starting point.

\section{Cosmological evolution induced by matter on the branes}

In this section we will construct solutions for $\alpha, \nu, \beta$ and $\varphi$ in the presence of matter on the branes. Matter on the branes contributes to the junction conditions (2.10) which affects, by continuity in $y$, the solutions across the bulk for all $y$. We will assume that the "amount" of matter is small compared to the fundamental scales $M_{11}, M_{G U T}$ (which are quite close to each other) and $M_{5}$, i.e.

$$
\rho^{(n)} \sim p^{(n)} \sim \mathcal{L}_{\text {matter }}^{(n)} \ll \gamma \kappa_{5}^{-2}
$$

On the other hand we will need no assumption on $\gamma R_{5}$, since we will obtain solutions exact in $y$.

First we recall that, in the case of an "empty" bulk, the Hubble constant $H$ was shown to satisfy $H \sim \rho$ [16], i.e. time derivatives $\partial_{t}$ are of the order $\partial_{t} \sim \mathcal{O}(\rho)$. A corresponding result was also obtained in 24] in the case where $\rho$ represents a $\varphi$ 
independent potential and $\rho R_{5} \kappa_{5}^{2} \gg 1$. Conventional cosmology, on the other hand, corresponds to $H^{2} \sim \rho$, i.e. $\partial_{t} \sim \mathcal{O}(\sqrt{\rho})$. This latter behaviour can be obtained in the case of additional cosmological constants in the bulk and on the branes $[17$ 23], and we will also obtain corresponding solutions in the present case.

Our ansatz for solutions in the presence of matter on the branes will be generalizations of the static solutions (2.12) in two respects: First, we add $y$ and $t$ dependent contributions to $\widetilde{\alpha}, \widetilde{\nu}, \widetilde{\beta}$ and $\widetilde{\varphi}$, which are of $\mathcal{O}\left(\rho \kappa_{5}^{2} \gamma^{-1}\right)$ where $\rho$ denotes the order of magnitude of all terms on the left-hand side of eq. (3.1). Second, we promote the constants $A, B$ and $C$ in (2.12) to time dependent parameters assuming, however, that all time derivatives are of the order

$$
\partial_{t} \sim \mathcal{O}\left(\left(\rho \gamma \kappa_{5}^{2}\right)^{1 / 2}\right)
$$

or smaller. (This ansatz allows, of course, for a still "weaker" time dependence with $\left.\partial_{t} \sim \mathcal{O}(\rho)\right)$. Thus we write

$$
\begin{gathered}
\alpha(y, t)=\frac{1}{2} \ln H(y, t)+\alpha_{0}(t)+\bar{\alpha}(y, t), \\
\nu(y, t)=\frac{1}{2} \ln H(y, t)+\nu_{0}(t)+\bar{\nu}(y, t), \\
\beta(y, t)=2 \ln H(y, t)+\beta_{0}(t)+\bar{\beta}(y, t), \\
\varphi(y, t)=\frac{3}{2} \ln H(y, t)+\varphi_{0}(t)+\bar{\varphi}(y, t), \\
\beta_{0}(t)=2 \varphi_{0}(t), \\
H(y, t)=\frac{\gamma}{3}|y|+C(t),
\end{gathered}
$$

with

$$
\bar{\alpha}, \bar{\nu}, \bar{\beta}, \bar{\varphi} \sim \mathcal{O}\left(\rho \kappa_{5}^{2} \gamma^{-1}\right)
$$

Equation (3.38) can be obtained from the dominant terms of the Einstein equations, and agrees with the static limit (2.12) after $B \rightarrow 2 \varphi_{0}(t)$. 
Let us first use this ansatz in the (05) component of the Einstein equations (2.30). Subsequently we replace $\beta_{0}$ by $2 \varphi_{0}$ everywhere according to eq. (3.3e), which simplifies several expressions. Using (3.2), (3.4), and keeping all terms up to $\mathcal{O}\left(\rho^{3 / 2}\right)$, eq. (2.30) can be brought into the form (with (2.5d) for $T_{05}$ )

$$
\begin{aligned}
& \left(\dot{\alpha}_{0}+\frac{\dot{C}}{2 H}\right)\left(\bar{\nu}^{\prime}-\bar{\alpha}^{\prime}\right)-\dot{\bar{\alpha}}^{\prime}+\left(2 \dot{\varphi}_{0}+3 \frac{\dot{C}}{H}\right)\left(\bar{\alpha}^{\prime}-\frac{\bar{\varphi}^{\prime}}{3}\right) \\
& +\frac{\gamma}{6 H}(\dot{\bar{\beta}}-2 \dot{\bar{\varphi}})+\frac{\gamma}{6 H^{2}} \dot{C}=0 .
\end{aligned}
$$

One notes that all terms are of $\mathcal{O}\left(\rho^{3 / 2}\right)$, except for the last term on the left-hand side, which is a priori (from $(3.2)$ ) of $\mathcal{O}\left(\rho^{1 / 2}\right)$. Thus $\dot{C}$ is exceptionally at most of $\mathcal{O}\left(\rho^{3 / 2}\right)$, and will not contribute to the dominant orders in $\rho$ in the following equations.

Next we insert our ansatz into the remaining Einstein equations (2.3) as well as (2.6). We use eqs. (2.5) for the components of the energy momentum tensor, and expand each expression in $\rho$ using (3.2) and (3.4). The dominant terms of $\mathcal{O}\left(\rho^{0}\right)$ cancel as they should, and subsequently we display all terms of $\mathcal{O}(\rho)$. The subsequent equations follow from eqs. (2.3a), (2.3b), (2.3d) and (2.6), after moving all time derivatives to the left (and with $\beta_{0}=2 \varphi_{0}$ ):

$$
\begin{aligned}
& \dot{\alpha}_{0}^{2}+2 \dot{\alpha}_{0} \dot{\varphi}_{0}-\frac{1}{3} \dot{\varphi}_{0}^{2}=H^{-3} e^{2 \nu_{0}-4 \varphi_{0}}\left(\bar{\alpha}^{\prime \prime}+\frac{\gamma}{6 H}\left(2 \bar{\varphi}^{\prime}-\bar{\beta}^{\prime}\right)\right. \\
& \left.+\frac{\gamma^{2}}{18 H^{2}}(\bar{\beta}-2 \bar{\varphi})\right) \\
& 2 \ddot{\alpha}_{0}+3 \dot{\alpha}_{0}^{2}-2 \dot{\nu}_{0}\left(\dot{\alpha}_{0}+\dot{\varphi}_{0}\right)+4 \dot{\alpha}_{0} \dot{\varphi}_{0}+2 \ddot{\varphi}_{0}+5 \dot{\varphi}_{0}^{2} \\
& =H^{-3} e^{2 \nu_{0}-4 \varphi_{0}}\left(2 \bar{\alpha}^{\prime \prime}+\bar{\nu}^{\prime \prime}+\frac{\gamma}{2 H}\left(2 \bar{\varphi}^{\prime}-\bar{\beta}^{\prime}\right)+\frac{\gamma^{2}}{6 H^{2}}(\bar{\beta}-2 \bar{\varphi})\right), \\
& \ddot{\alpha}_{0}+2 \dot{\alpha}_{0}^{2}-\dot{\alpha}_{0} \dot{\nu}_{0}+\frac{1}{3} \dot{\varphi}_{0}^{2}=H^{-3} e^{2 \nu_{0}-4 \varphi_{0}}\left(\frac{\gamma}{6 H}\left(3 \bar{\alpha}^{\prime}+\bar{\nu}^{\prime}-2 \bar{\varphi}^{\prime}\right)\right. \\
& \left.+\frac{\gamma^{2}}{18 H^{2}}(\bar{\beta}-2 \bar{\varphi})\right), \\
& \ddot{\varphi}_{0}+\dot{\varphi}_{0}\left(3 \dot{\alpha}_{0}-\dot{\nu}_{0}+2 \dot{\varphi}_{0}\right)=H^{-3} e^{2 \nu_{0}-4 \varphi_{0}}\left(\bar{\varphi}^{\prime \prime}+\frac{\gamma}{2 H}\left(3 \bar{\alpha}^{\prime}+\bar{\nu}^{\prime}-\bar{\beta}^{\prime}\right)\right. \\
& \left.+\frac{\gamma^{2}}{3 H^{2}}(\bar{\beta}-2 \bar{\varphi})\right) \cdot
\end{aligned}
$$


These four equations fix the $y$ dependence of $\bar{\alpha}, \bar{\nu}, \bar{\beta}$ and $\bar{\varphi}$ :

$$
\begin{gathered}
\bar{\alpha}(y, t)=\widehat{\alpha}(t) H^{5}+\frac{1}{3} F(y), \\
\bar{\nu}(y, t)=\widehat{\nu}(t) H^{5}+\frac{1}{3} F(y), \\
\bar{\beta}(y, t)=\widehat{\beta}(t) H^{5}+2 F(y)+\frac{2 H}{\gamma} F^{\prime}(y), \\
\bar{\varphi}(y, t)=\widehat{\varphi}(t) H^{5}+F(y)
\end{gathered}
$$

with $H$ as in eq. (2.12d) and $F(y)$ arbitrary.

The four time-dependent parameters $\widehat{\alpha}, \widehat{\nu}, \widehat{\beta}$ and $\widehat{\varphi}$ are constrained by the three junction conditions (2.10) in terms of the matter on the branes. Plugging our ansätz (3.3) as well as (3.7) into eqs. (2.10), one finds again that the leading terms of $\mathcal{O}\left(\rho^{0}\right)$ cancel, and the terms of $\mathcal{O}(\rho)$ can be brought into the form

$$
\begin{gathered}
10 \gamma \varepsilon^{(n)} \widehat{\alpha}=-\left(H^{(n)}\right)^{-2} \kappa_{5}^{2} \rho^{(n)} e^{2 \varphi_{0}}+\gamma(\widehat{\beta}-2 \widehat{\varphi}), \\
10 \gamma \varepsilon^{(n)} \widehat{\nu}=\left(H^{(n)}\right)^{-2} \kappa_{5}^{2}\left(3 p^{(n)}+2 \rho^{(n)}\right) e^{2 \varphi_{0}}+\gamma(\widehat{\beta}-2 \widehat{\varphi}), \\
10 \gamma \varepsilon^{(n)} \widehat{\varphi}=-\frac{3}{2}\left(H^{(n)}\right)^{-2} \kappa_{5}^{2} \frac{\delta \mathcal{L}_{\text {matter }}^{(n)}}{\delta \varphi} e^{2 \varphi_{0}}+3 \gamma(\widehat{\beta}-2 \widehat{\varphi}),
\end{gathered}
$$

where $H^{(n)}$ denotes $H\left(y^{(n)}\right)$ with $H(y)$ as in eq. (2.12d), and $\varepsilon^{(n)}=+1$ for $y^{(n)}=0$, $\varepsilon^{(n)}=-1$ for $y^{(n)}=\pi R_{5}$. The dependence on the function $F(y)$ of eqs. (3.7) cancels in the junction conditions (2.10).

Altogether we thus have six equations, three junction conditions at the brane $n=1$, and three junction conditions at the brane $n=2$. Let us first discuss, to what extent these six equations restrict the properties of the matter on the different branes. We recall that, e.g., in the case of an "empty" bulk considered in [16], $\rho^{(2)}$ is fixed in terms of $\rho^{(1)}$, and $p^{(2)}$ in terms of $p^{(1)}$. In the present case the three equations which involve the properties of the matter on the brane 2 can be brought into the form 


$$
\begin{gathered}
\left(H^{(1)}\right)^{2} p^{(1)}+\left(H^{(2)}\right)^{2} p^{(2)}=-\left(H^{(1)}\right)^{2} \rho^{(1)}-\left(H^{(2)}\right)^{2} \rho^{(2)} \\
\left(H^{(1)}\right)^{2} \frac{\delta \mathcal{L}_{\text {matter }}^{(1)}}{\delta \varphi}+\left(H^{(2)}\right)^{2} \frac{\delta \mathcal{L}_{\text {matter }}^{(2)}}{\delta \varphi}=2\left(H^{(1)}\right)^{2} \rho^{(1)}+2\left(H^{(2)}\right)^{2} \rho^{(2)}, \\
\gamma \kappa_{5}^{2}\left[\left(H^{(1)}\right)^{2} \rho^{(1)}+\left(H^{(2)}\right)^{2} \rho^{(2)}\right]=2(\widehat{\beta}-2 \widehat{\varphi}) .
\end{gathered}
$$

At this stage we have to recall that $H$, defined in eq. 2.12d), depends on an as yet arbitrary parameter $C$ (with negligible time dependence): we have $H^{(1)}=C$, $H^{(2)}=\frac{\gamma}{3} \pi R_{5}+C$. Hence eqs. (3.9) do not necessarily constrain the matter on the brane 2 in terms of the matter on the brane 1 , but can rather serve to fix $C$.

Of particular interest is the case where the matter fields $\phi_{i}$ on the branes are constant (at the minima of their potentials), and the only role of $\mathcal{L}_{\text {matter }}^{(n)}$ is thus to provide additional (possibly constant) potentials for the modulus field $\varphi$ on the branes. Then we can write

$$
\begin{gathered}
\mathcal{L}_{\text {matter }}^{(n)}=-\widehat{V}^{(n)}(\varphi) \\
\rho^{(n)}=\widehat{V}^{(n)}(\varphi) \\
p^{(n)}=-\widehat{V}^{(n)}(\varphi) .
\end{gathered}
$$

Hence eq. (3.9a is satisfied identically. Eq. (3.9b) serves to fix $C$ in terms of the potentials on the branes and $\varphi$. However, in order not to contradict the previous result $\dot{C} \lesssim \mathcal{O}\left(\rho^{3 / 2}\right)$, one must have either $\dot{\varphi}_{0} \sim 0$, or $\widehat{V}^{(1)}(\varphi)=$ const. $\widehat{V}^{(2)}(\varphi)$. Eq. (3.90) fixes the combination $(\widehat{\beta}-2 \widehat{\varphi})$.

Herewith we conclude the discussion on the relation between the matter on the different branes, and concentrate subsequently on the physics on brane 1 assuming that eqs. (3.9) are satisfied. Note, however, that the two branes are physically distinct in Hořava-Witten theory, since the dominant potentials $V^{(n)}(\varphi)$ on the branes differ in their sign (cf. eq. (2.8)). On the other hand we find from eqs. (3.8) that we can exchange the role of the two branes by changing the sign of $\gamma$, and redefining the parameter $\widehat{\beta}$ : 


$$
\gamma \rightarrow-\gamma, \widehat{\beta} \rightarrow 4 \widehat{\varphi}-\widehat{\beta}
$$

In order to solve eqs. (3.8) on brane 1 it is convenient to define the parameters $w, d$ as follows:

$$
\begin{gathered}
p^{(1)}=w \rho^{(1)}, \\
\frac{\delta \mathcal{L}_{\text {matter }}^{(1)}}{\delta \varphi}=-d \rho^{(1)} .
\end{gathered}
$$

In general the parameter $d$ will depend on $\varphi$, unless $\mathcal{L}_{\text {matter }}^{(1)}$ and $\rho^{(1)}, p^{(1)}$ happen to be related as, e.g., in eqs. (3.10) with

$$
\widehat{V}^{(n)}(\varphi) \sim e^{d \varphi} .
$$

With eqs. (3.12) and $H^{(1)}=C$, the general solution of eqs. (3.8) on brane 1 can be written as

$$
\begin{gathered}
\widehat{\alpha}=\frac{1}{16} \widehat{\beta}-\frac{\kappa_{5}^{2} \rho^{(1)} e^{2 \varphi_{0}}}{160 \gamma C^{2}}(3 d+16), \\
\widehat{\nu}=\frac{1}{16} \widehat{\beta}+\frac{\kappa_{5}^{2} \rho^{(1)} e^{2 \varphi_{0}}}{160 \gamma C^{2}}(-3 d+16(3 w+2)), \\
\widehat{\varphi}=\frac{3}{16} \widehat{\beta}+\frac{3 \kappa_{5}^{2} \rho^{(1)} e^{2 \varphi_{0}}}{32 \gamma C^{2}} d,
\end{gathered}
$$

with $\widehat{\beta}$ arbitrary. (Eventually $\widehat{\beta}$ can be fixed, combining eqs. (3.14d) and (3.9d), in terms of $\left.\rho^{(2)}\right)$.

Combining eqs. (3.14) and eqs. (3.7) we have thus obtained the general solutions for $\bar{\alpha}, \bar{\nu}, \bar{\beta}, \bar{\varphi}$ in terms of the properties of the matter on the branes and for all values of $y$.

Let us recall the ansatz (3.3) for the fields $\alpha, \nu, \beta$ and $\varphi$. Since we obtained $\dot{C} \lesssim \mathcal{O}\left(\rho^{3 / 2}\right)$, the dominant contributions to these fields decompose into a sum of $y$ dependent and $t$ dependent terms. The cosmological evolution on any brane is thus 
determined by $\alpha_{0}(t), \nu_{0}(t)$ and $\varphi_{0}(t)$. The knowledge of the subdominant contributions $\bar{\alpha}, \bar{\nu}, \bar{\beta}$ and $\bar{\varphi}$ to eqs. (3.3) is required in order to obtain the $t$ dependence of $\alpha_{0}, \nu_{0}$ and $\varphi_{0}$ from eqs. (3.6), which we exploit in the following.

Once we plug our solutions (3.7) for $\bar{\alpha}, \bar{\nu}, \bar{\beta}$ and $\bar{\varphi}$, together with eqs. (3.14), into eqs. (3.6), we first observe that all dependence on the arbitrary function $F(y)$ in (3.7) as well as on $\widehat{\beta}$ cancels out. Hence the time dependence of $\alpha_{0}, \nu_{0}$ and $\varphi_{0}$ can be related to the properties of the matter on the brane 1 only, which are encoded in our parameters $\rho^{(1)}, w$ and $d$. Second, we may choose the gauge $\nu_{0}(t)=0$, such that $t$ is proportional - up to small corrections - to the cosmic time: now we have $\partial_{t} \nu(y, t) \lesssim \mathcal{O}\left(\rho^{3 / 2}\right)$ (cf. eq. 3.3b), since $\dot{C}$ and $\dot{\bar{\nu}}$ are at most of $\mathcal{O}\left(\rho^{3 / 2}\right)$.

Since one finds that one of eqs. (3.6), e.g. eq. (3.6b), is redundant, we just give our results for eqs. (3.6a), (3.60) and (3.6d):

$$
\begin{gathered}
\dot{\alpha}_{0}^{2}+2 \dot{\alpha}_{0} \dot{\varphi}_{0}-\frac{1}{3} \dot{\varphi}_{0}^{2}=-\frac{2}{9} \frac{\gamma \kappa_{5}^{2} \rho^{(1)}}{C^{2}} e^{-2 \varphi_{0}} \\
\ddot{\alpha}_{0}+2 \dot{\alpha}_{0}^{2}+\frac{1}{3} \dot{\varphi}_{0}^{2}=\frac{\gamma \kappa_{5}^{2} \rho^{(1)}}{36 C^{2}} e^{-2 \varphi_{0}}(3 w-1-3 d), \\
\ddot{\varphi}_{0}+\dot{\varphi}_{0}\left(3 \dot{\alpha}_{0}+2 \dot{\varphi}_{0}\right)=\frac{\gamma \kappa_{5}^{2} \rho^{(1)}}{12 C^{2}} e^{-2 \varphi_{0}}(3 w-1+d) .
\end{gathered}
$$

Eqs. (3.15) justify, a posteriori, our initial ansatz (3.2) for the order of $\partial_{t}$, provided $C^{2} \exp \left(2 \varphi_{0}\right) \sim \mathcal{O}(1)$.

Taking the time derivative of eq. (3.15a) and using all eqs. (3.15), the analog of the standard energy conservation condition can be obtained:

$$
\dot{\rho}^{(1)}=-3 \rho^{(1)} \dot{\alpha}_{0}(1+w)+d \rho^{(1)} \dot{\varphi}_{0} .
$$

First, if we insert our solution (3.7) for $\bar{\alpha}, \bar{\nu}, \bar{\beta}$ and $\bar{\varphi}$, together with eqs. (3.14) and eq. (3.16), into eq. (3.5), we obtain a trivial time dependence of $C$ :

$$
\dot{C}=0
$$

Second, eq. (3.16) differs from the standard condition for energy conservation due to the last term. This term describes the "disappearance" of energy into the fifth dimension, if both $\delta \mathcal{L}_{\text {matter }}^{(1)} / \delta \varphi \sim d \neq 0$ and $\dot{\varphi}_{0} \neq 0$. In fact, if $\mathcal{L}_{\text {matter }}^{(1)}$ is of the 
form of a potential $-\widehat{V}^{(1)}(\varphi)$ (as in eqs. (3.10) ) we have $w=-1$ and, using $\dot{\varphi} \sim \dot{\varphi}_{0}$, eq. (3.10b) and eq. (3.12b),

$$
\dot{\rho}^{(1)}=\partial_{t} \widehat{V}^{(1)}(\varphi)=\frac{\delta \widehat{V}^{(1)}}{\delta \varphi} \dot{\varphi} \cong-\frac{\delta \mathcal{L}_{\text {matter }}^{(1)}}{\delta \varphi} \dot{\varphi}_{0}=d \rho^{(1)} \dot{\varphi}_{0}
$$

in agreement with $(3.16)$.

For $d \neq 0$, a "standard" cosmological evolution on any brane, which requires standard energy conservation, is only possible for $\dot{\varphi}_{0}=\dot{\beta}_{0}=0$. From eq. (3.15d) this situation is feasable only for

$$
d=1-3 w,
$$

which requires a particular dependence of $\mathcal{L}_{\text {matter }}^{(1)}$ on $\varphi$. If, again, $\mathcal{L}_{\text {matter }}^{(1)}$ is of the form of a potential (hence $w=-1$ ), we need $d=4$ or

$$
\widehat{V}^{(1)}(\varphi)=\text { const. } e^{4 \varphi}
$$

Furthermore, from eq. (3.15a), $\dot{\varphi}_{0}=0$ implies immediately

$$
\gamma \cdot \rho^{(1)} \leq 0
$$

Thus, if we want to accomodate a standard positive energy density $\rho^{(1)}$, we have to choose $\gamma<0$, i.e. brane 1 has to be the one with a positive tension (since now the dominant contribution $V^{(1)}(\varphi)$, from eq. (2.8), is positive). This result coincides with the one obtained in the case of cosmological constants in the bulk and on the branes $[17-23]$.

If eq. (3.19) is satisfied, eqs. (3.15) allow for $\dot{\varphi}_{0}=0$. Since we have $\dot{\alpha} \sim \dot{\alpha}_{0}$ and, from our definition (2.2) of the metric, $\dot{\alpha}$ corresponds to the Hubble parameter, eqs. (3.15a) and (3.15b) are easily seen to correspond to the ordinary Freedman equations for $\gamma<0$. (A constant term in $\nu$ allows for a constant rescaling of $t$ such that the right-hand sides of eqs. (3.15a) and (3.15b), for $\dot{\varphi}_{0}=0$, can always be brought into standard form).

If eq. (3.19) is not satisfied, we have necessarily $\dot{\varphi}_{0} \neq 0$ and, from eq. (3.3e), $\dot{\beta}_{0} \neq 0$, i.e. solutions corresponding to a "rolling radius": the physical distance between the two branes, given by $\int e^{\beta(y)} d y$ with $y=\left\{0, \pi R_{5}\right\}$, varies with $t$.

Explicit solutions to eqs. (3.15) can be given if $w=$ const. and $d=$ const. From eq. (3.12b) the second condition is satisfied if 


$$
\mathcal{L}_{\text {matter }}^{(1)}(\varphi) \sim \rho^{(1)}(\varphi, t)=\widehat{\rho}^{(1)}(t) e^{d \varphi}
$$

Then eqs. (3.15) are solved for

$$
\begin{aligned}
& \alpha_{0}(t)=\text { const. }+r \ln t, \varphi_{0}(t)=\text { const. }+s \ln t, \\
& \rho^{(1)} \sim \text { const. } t^{2 s-2} \quad\left(\text { or } \hat{\rho}^{(1)} \sim \text { const. } t^{(2-d) s-2}\right),
\end{aligned}
$$

with

$$
r=\frac{2(w-3-d)}{3 w^{2}-6 w d-d^{2}-11}, s=\frac{2(3 w-1+d)}{3 w^{2}-6 w d-d^{2}-11},
$$

provided the denominators are non-zero. In the case where $\mathcal{L}_{\text {matter }}^{(1)}(\varphi)$ is just an additional potential in $\varphi$, as in eqs. (3.10) with $n=1$, we have $w=-1$ and thus

$$
r=\frac{2(4+d)}{(4-d)(2-d)}, s=\frac{2}{2-d} \quad \text { if } d \neq 2,4 \text {. }
$$

(The particular case $d=-2$, where $\widehat{V}^{(n)}(\varphi)$ in eqs. (3.10) has the same functional dependence on $\varphi$ as $V^{(n)}(\varphi)$ in eq. (2.8), has already been considered in [12].) For $d=4$ we are back in the situation where eq. (3.19) is valid (and where standard inflation on the branes is obtained for $w=-1$ ), whereas for $d=2$ we obtain inflationary evolution both on the branes and across the bulk, i.e. in $\beta(t) \sim 2 \varphi_{0}(t)$ :

$$
\begin{aligned}
& a(t)=e^{\alpha(t)} \sim e^{\alpha_{0}(t)} \sim e^{\text {const. } t} \\
& \dot{\varphi}_{0}=\frac{1}{3} \dot{\alpha}_{0}, \rho(t)=\bar{\rho} e^{2 \varphi_{0}(t)}
\end{aligned}
$$

with $\dot{\bar{\rho}}=0$, and const. $\sim \pm \sqrt{\bar{\rho}}$.

Herewith we conclude the different solutions to eqs. (3.15), which will be discussed in the next section.

\section{Discussion and conclusions}

In the previous section we have constructed various cosmological solutions in (compactified) Hořava-Witten theory with additional matter on the branes, assuming $\rho \ll \gamma \kappa_{5}^{-2}$, cf. eq. (3.1). Actually, whenever $\rho$ varies with $t$, this assumption 
restricts the validity of the solutions to corresponding regimes in $t$, typically to sufficiently late time once $\rho$ decays in $t$.

Generally compactified Hořava-Witten theory suffers from the usual moduli problem, i.e. there are scalar degrees of freedom with vanishing potentials. In the present framework this phenomenon corresponds to the presence of arbitrary constants in the static solutions (2.12) [4] (in the absence of additional matter), and to the existence of time dependent solutions with $\partial_{t} \sim \mathcal{O}(\gamma)$ [9, 10]. Since we assumed a much weaker time dependence, cf. eq. (3.2), our solutions require particular initial conditions in the form of nearly static (and homogeneous) configurations. However, once matter on the branes is present, the fields cannot vary slower with $t$ than indicated by our solutions.

One aspect of the moduli problem is the fact that generically the physical distance between the branes, i.e. the $y y$ component of the metric (parametrized by $\beta$ ), varies in time. Our results show that, once matter is present only on the branes, this phenomenon is unavoidable, unless the matter couples to the universal Calabi-Yau modulus field $\varphi$ in a particular way, cf. eq. (3.19). Possibly the five dimensional radius can be stabilized by means of an additional potential for $\varphi$ in the bulk $[23,25$ - 30]. Then our cosmological solutions would be relevant at times $t$, at which potentials $V^{(n)}(\varphi) \ll \gamma \kappa_{5}^{-2}$ on the branes are present (e.g. due to gaugino condensation), but where additional bulk-potentials are not yet switched on.

Let us summarize our results in the case where the additional matter Lagrangian on the branes corresponds to potentials for $\varphi$, i.e. where the equation of state corresponds to $w=-1$. Exact solutions have been obtained for potentials of the form

$$
\widehat{V}^{(n)}(\varphi)\left(=-\rho^{(n)}\right)=\widehat{\rho}^{(n)} e^{d \varphi}
$$

Generically we obtain solutions with a power law behaviour in $t$ for the scale factor $a$ and the physical distance $R_{\text {phys }}$ between the branes (cf. eqs. (3.23) and below; note that $\widehat{\rho}^{(n)}$ is time independent for $w=-1$, where eqs. (3.25) are valid):

$$
\begin{gathered}
a(y, t)=e^{\alpha(y, t)} \sim \operatorname{const.}(y) e^{\alpha_{0}(t)} \sim t^{r}, \\
R_{\text {phys }}(t) \sim e^{\beta_{0}(t)}=e^{2 \varphi_{0}(t)} \sim t^{2 s}
\end{gathered}
$$


with $r, s$ as in eqs. (3.25) (for $d=0$, e.g., one has $r=s=1$ ). In the particular case $d=2$ one obtains, from eqs. (3.26), inflationary (i.e. exponentially increasing or decreasing) evolutions for $a, R_{\text {phys }}$ and $\widehat{\rho}^{(n)}$. In the other particular case $d=4$ the ordinary Freedman equations hold on the branes: Now $R_{\text {phys }}$ can be time independent (since $\dot{\beta}_{0}=2 \dot{\varphi}_{0}=0$ ), and inflationary evolution happens only "parallel" to the branes in terms of an exponential $t$ dependence of $a$.

Once the equation of state of the matter on the branes differs from $w=-1$ we can still obtain solutions with constant $R_{\text {phys }}$, if eq. (3.19) is satisfied. In these cases the cosmological evolution is of the standard form. For radiation dominated matter $\left(w=\frac{1}{3}\right)$, e.g., standard cosmological evolution is obtained for $d=0$, i.e. when the (dominant part of the) matter action is independent of $\varphi$. For nonrelativistic matter $(w=0)$ standard cosmological evolution is obtained once the matter action satifies eq. (3.12b) with $d=1$. It remains to be seen whether these solutions constitute an alternative to the (presently ad hoc) radius fixation by potentials in the bulk, i.e. whether corresponding couplings of the Calabi-Yau modulus $\varphi$ to matter on the branes and sufficiently well-behaved initial conditions can be obtained.

In any case we have seen that matter induced cosmological evolution in HořavaWitten theory (albeit in its simplest version with just the universal Calabi-Yau modulus in the bulk) differs considerably from simpler scenarios as an empty bulk or a cosmological constant in the bulk. 


\section{References}

[1] P. Hořava and E. Witten, hep-th/9510209, Nucl. Phys. B460 (1996) 506; hepth/9603142, Nucl. Phys. B475 (1996) 94.

[2] E. Witten, hep-th/9602070, Nucl. Phys. B471 (1996) 135.

[3] T. Banks, M. Dine, hep-th/9605136, Nucl. Phys. B479 (1996) 173.

[4] A. Lukas, B.A. Ovrut, K. Stelle, D. Waldram, hep-th/9803235, Phys. Rev. D59 (1999) 086001.

[5] J. Ellis, Z. Lalak, S. Pokorski, W. Pokorski, hep-ph/9805377, Nucl. Phys. B540 (1999) 149.

[6] A. Lukas, B.A. Ovrut, K. Stelle, D. Waldram, hep-th/9806051, Nucl. Phys. B552 (1999) 246.

[7] J. Ellis, Z. Lalak, W. Pokorski, hep-th/9811133.

[8] K. Benakli, hep-th/9804096, Int. J. Mod. Phys. D8 (1999) 153.

[9] A. Lukas, B.A. Ovrut, D. Waldram, hep-th/9806022, Phys. Rev. D60 (1999) 086001.

[10] H. Reall, hep-th/9809195, Phys. Rev. D59 (1999) 103506.

[11] H. Chamblin, H. Reall, hep-th/9903225.

[12] J. Lidsey, gr-qc/9911066.

[13] L. Randall, R. Sundrum, hep-ph/9905221, Phys. Rev. Lett. 83 (1999) 3370.

[14] M. Gogberashvili, hep-ph/9904383, Mod. Phys. Lett. A14 (1999) 2025.

[15] L. Randall, R. Sundrum, hep-th/9906064, Phys. Rev. Lett. 83 (1999) 4670.

[16] P. Binétruy, C. Deffayet, D. Langlois, hep-th/9905012.

[17] C. Csaki, M. Graesser, C. Kolda, J. Terning, hep-ph/9906513, Phys. Lett. B462 (1999) 34 . 
[18] J. Cline, C. Grojean, G. Servant, hep-ph/9906523, Phys. Rev. Lett. 83 (1999) 4245.

[19] P. Kanti, I.I. Kogan, K.A. Olive, M.Pospelov, hep-ph/9909481, Phys. Lett. B468 (1999) 31.

[20] T. Shiromizu, K.-I. Maeda, M. Sasaki, gr-qc/9910076.

[21] E. Flanagan, S.-H. Tye, I. Wasserman, hep-ph/9910498.

[22] P. Binétruy, C. Deffayet, U. Ellwanger, D. Langlois, hep-th/9910219.

[23] C. Csaki, M. Graesser, L. Randall, J. Terning, hep-ph/9911406.

[24] A. Lukas, B.A. Ovrut, D. Waldram, hep-th/9902071, Phys. Rev. D61 (2000) 023506 .

[25] A. Lukas, B.A. Ovrut, D. Waldram, hep-th/9611204, Nucl. Phys. B509 (1998) 169.

[26] K. Choi, H.B. Kim, H. Kim, hep-th/9808122, Mod. Phys. Lett. A14 (1999) 125.

[27] W. Goldberger, M. Wise, hep-ph/9907447, Phys. Rev. Lett. 83 (1999) 4922.

[28] O. De Wolfe, D. Freedman, S. Gubser, A. Karch, hep-th/9909134.

[29] M. Luty, R. Sundrum, hep-th/9910202.

[30] P. Kanti, I.I. Kogan, K.A. Olive, M. Pospelov, hep-ph/9912266. 